PROCEEDINGS OF THE

AMERICAN MATHEMATICAL SOCIETY

Volume 134, Number 3, Pages 893-895

S 0002-9939(05)08056-

Article electronically published on July 8, 2005

\title{
MAPS INTO COMPLEX SPACE
}

\author{
HOWARD JACOBOWITZ
}

(Communicated by Jozef Dodziuk)

\begin{abstract}
If the dimension of $M$ is denoted by $2 k-1$ or $2 k$, then a generic map $F: M \rightarrow C^{k}$ satisfies $d F_{1} \wedge \ldots \wedge d F_{k} \neq 0$, while in certain cases there is no map $F: M \rightarrow C^{k+1}$ that satisfies $d F_{1} \wedge \ldots \wedge d F_{k+1} \neq 0$.
\end{abstract}

\section{Statement of Results}

Let $F: M \rightarrow R^{2}$ be any map. The components of $F$ may be approximated by Morse functions. From this, we see that the rank of the Jacobian of a generic map of $M \rightarrow R^{2}$ is everywhere greater than zero. This can be reformulated as: A generic map $F: M^{n} \rightarrow C^{1}$ has, at all points, $d F \neq 0$. This suggests the question: For a given value of $n$, what is the largest value of $r$ for which every manifold of dimension $n$ has a map $F=\left(F_{1}, \ldots F_{r}\right)$ into $C^{r}$ with $d F_{1} \wedge \ldots \wedge d F_{r}$ never zero?

Given $n$, set $r=\left[\left[\frac{n}{2}\right]\right]$, the smallest integer greater than or equal to $\frac{n}{2}$.

Theorem 1.1. For every manifold $M^{n}$ of dimension $n$ there is a generic set of maps $F: M^{n} \rightarrow C^{r}$ with $d F_{1} \wedge \ldots \wedge d F_{r}$ never equal to zero.

By a generic set of functions or maps on a compact manifold we mean a set that is open in the $C^{1}$ topology and dense in the $C^{\infty}$ topology; on a non-compact manifold we mean the countable intersection of such sets. Note that the theorem states that $M^{n}$ can be mapped into $R^{k}, k \leq n$ for $n$ even and $k \leq n+1$ for $n$ odd, in a special way.

Corollary 1. Every manifold $M^{n}$ of dimension $n$ admits an involutive sub-bundle of rank $k$ for each $k, k \geq\left[\frac{n}{2}\right]$.

A bundle $V \subset C T M$ is involutive if the Lie bracket condition $[V, V] \subset V$ holds for all local sections. This is an interesting restriction, motivated by partial differential equations and generalizing the Frobenius condition for sub-bundles of $T M$. Here $\left[\frac{n}{2}\right]$ is the largest integer less than or equal to $\frac{n}{2}$ and so $n=\left[\frac{n}{2}\right]+\left[\left[\frac{n}{2}\right]\right]$. The corollary follows from the theorem by taking the annihilator of $\left\{d F_{1}, \ldots, d F_{r}\right\}$.

The value for $r$ is sharp (for $n=4 k$ ) if we insist that the same $r$ works for all manifolds of a fixed dimension. The basic idea is that every complex bundle of rank $N$ over $M^{n}, N>\frac{n}{2}$, admits $N-\left[\frac{n}{2}\right]$ independent global sections. The rank of $C T^{*} M^{n}$ is $n$ and $d F_{1}, \ldots, d F_{r}$ provide $r$ independent global sections. Thus for $r>n-\left[\frac{n}{2}\right]=\left[\left[\frac{n}{2}\right]\right]$, we have a restriction on the bundle. Here is one simple way to formulate this restriction. Note that we use only the existence of the global

Received by the editors September 22, 2004.

2000 Mathematics Subject Classification. Primary 58A99; Secondary 58J10.

(C)2005 American Mathematical Society Reverts to public domain 28 years from publication 
section and ignore the more refined information that each section is given by a closed one-form.

Theorem 1.2. Let $F: M^{4 k} \rightarrow C^{2 k+1}$ satisfy $d F_{1} \wedge \ldots \wedge d F_{2 k+1}$ never equal to zero. Then the top Pontryagin class of $M$ is zero.

Since there exist manifolds $M^{4 k}$ with $P_{k} \neq 0, r=2 k$ is the largest $r$ that works for all manifolds of dimension $4 k$.

Examples. There exists a map $F: C P^{2} \rightarrow C^{2}$ with $d F_{1} \wedge d F_{2}$ never equal to zero; any map $F: C P^{2} \rightarrow C^{3}$ has $d F_{1} \wedge d F_{2} \wedge d F_{3}$ somewhere equal to zero. Whether there exists a map of $C P^{1} \rightarrow C^{2}$ with $d F_{1} \wedge d F_{2} \neq 0$ is left open by these theorems. Note that the existence of such a map implies the (true) statement that $C T S^{2}$ is trivial. There does exist such a map into $C^{1}$, and it can be obtained by projecting $S^{2}$ into any plane. Also note that the case $n=2$ is related to symplectic structures. Namely, identify $R^{4}$ with $C^{2}$ by using $z_{1}=x_{1}-i y_{1}$ and $z_{2}=x_{2}+i y_{2}$. Then $d z_{1} \wedge d z_{2}=\omega_{1}+\omega_{2}$ for symplectic forms $\omega_{1}$ and $\omega_{2}$, positive with respect to the orientation given by $\left(x_{1}, x_{2}, y_{1}, y_{2}\right)$. No symplectic structure on $R^{4}$ has a compact symplectic submanifold. So if $F: S^{2} \rightarrow C^{2}$, then $F^{*} \omega_{1}$ and $F^{*} \omega_{2}$ must have zeroes, and we are asking if $F$ can be chosen such that they have no common zeroes.

\section{Proof of Theorem 1.1}

We use notation and results from [1, Chapter 2. Let $X^{(1)}$ be the space of one jets of maps $F: M^{n} \rightarrow C^{r}$ written locally as $\left\{\left(p, F(p), d F_{1}(p), \ldots, d F_{r}(p)\right)\right\}$ and let $S$ be the subset given by $\left\{(p, c, \theta): \theta_{1} \wedge \ldots \wedge \theta_{r}=0\right\}$.

Lemma 2.1. $S$ is a stratified subset of $X^{(1)}$ of codimension $2(n+1-r)$.

To prove this, we need to show

$$
S=\bigcup_{i=0}^{N} S_{i},
$$

where each $S_{i}$ is a locally closed sub-manifold of $X^{(1)}$ satisfying

$$
\bar{S}_{j}=\bigcup_{i=j}^{N} S_{i},
$$

and

(2) $\operatorname{codim} S_{0}=2(n+1-r)$.

Proof. Let $S_{i}$ be the subset of $S$ defined by

$$
\left\{(p, c, \theta): \theta_{1} \wedge \ldots \wedge \theta_{r-i}=0 \text { and } \theta_{1} \wedge \ldots \wedge \theta_{r-i-1} \neq 0\right\} .
$$

So $N=r-1$ and $S_{N}=\left\{p, c, \theta: \theta_{1}=0\right\}$. Then the first condition is obvious. For the second, we count the equations that define $S_{0}$ near one of its points, $\left(p, c, \theta^{0}\right)$. We have that $(p, c, \theta) \in S_{0}$ provided $\theta_{r} \in \operatorname{span}\left\{\theta_{1}, \ldots, \theta_{r-1}\right\}$. Expressing $\theta_{r}$ as a linear combination with complex coefficients of basis elements $\left\{\theta_{1}, \ldots, \theta_{r-1}, e_{1}, \ldots, e_{n-r+1}\right\}$, we see that there are $n-r+1$ independent equations. This establishes the second condition. 
We now require $2(n+1-r)>n$. Thus the codimension of $S$ in $X^{(1)}$ is greater than the dimension of $M$. It follows from a basic result of differential topology that any map of $M$ into $X^{(1)}$ may be perturbed so as to not intersect $S$. The (simplest case of) the Thom Transversality Theorem is more precise. Any map of $M \rightarrow C^{r}$ may be perturbed to obtain a map of $M \rightarrow C^{r}$ whose one jet does not intersect $S$. Further, maps of this latter kind are generic. Finally, note that $2(n+1-r)>n$ is equivalent to $r \leq\left[\left[\frac{n}{2}\right]\right]$.

\section{Proof of Theorem 1.2}

Consider a map $F: M^{n} \rightarrow C^{r}$ with $d F_{1} \wedge \ldots \wedge d F_{r}$ never equal to zero. Let $V \subset C T M$ be the bundle whose fiber at $p$ is given by

$$
V=\left\{v: d F_{j}(v)=0 \text { at } p, \quad j=1, \ldots, r\right\}
$$

and let $\Omega \subset C T^{*} M$ be the span of $\left\{d F_{1}, \ldots, d F_{r}\right\}$. Use any hermitian metric to get a decomposition

$$
C T M=V \oplus Q
$$

Lemma 3.1. $\Omega \simeq Q^{*}$.

Proof. Recall that $Q^{*}$ is the set of linear functionals on the fibers of $Q$. Restricting an element $\theta \in \Omega$ to act on $Q$ produces an element $l_{\theta}$ of $Q^{*}$. The map $\theta \rightarrow l_{\theta}$ is injective and, since $\Omega$ and $Q^{*}$ have the same dimension, it is also surjective.

Each $d F_{j}$ is a global, non-zero section of $\Omega$. Thus $\Omega, Q^{*}$, and $Q$ are all trivial bundles. Thus, for the Chern class of $C T M$ we have, as in 2], page 164,

$$
c(C T M)=c(V \oplus Q)=c(V) \wedge c(Q)=c(V) .
$$

Now let $P_{k}(T M) \in H^{4 k}(M, Z)$ be the top Pontryagin class of $M$. We have from [2], page 174,

$$
P_{k}(T M)=(-1)^{k} c_{2 k}(C T M)=(-1)^{k} c_{2 k}(V) .
$$

In the case of Theorem $1.2 \operatorname{rankV}=4 k-(2 k+1)=2 k-1<2 k$ and so $P_{k}(T M)=0$.

\section{REFERENCES}

[1] Y. Eliashberg and N. Mishachev, Introduction to the h-Principle, American Mathematical Society, Providence, Rhode Island, 2002. MR.1909245 (2003g:53164)

[2] J. Milnor and J. Stasheff, Characteristic classes, Annals of Mathematics Studies, No. 76. Princeton University Press, Princeton, N. J., 1974. MR0440554 (55:13428)

Department of Mathematical Sciences, Rutgers University, Camden, New Jersey 08102

E-mail address: jacobowi@camden.rutgers.edu 